\title{
New exactly solvable periodic potentials for the Dirac equation
}

\author{
B F Samsonov $\dagger \ddagger \S$, A A Pecheritsin\|, E O Pozdeeva $\|$ and \\ M L Glasser ף \\ † Departamento de Física Teórica, Universidad de Valladolid, 47005 Valladolid, \\ Spain \\ II Department of Quantum Field Theory, Tomsk State University, Lenin Ave, \\ 634050 Tomsk, Russia \\ I Department of Physics and Center for Quantum Device Technology, Clarkson \\ University, Potsdam NY 13699-5820 (USA) \\ E-mail: samsonov@phys.tsu.ru, pecher@ido.tsu.ru, laryg@clarkson.edu
}

\begin{abstract}
A new exactly solvable relativistic periodic potential is obtained by the periodic extension of a well-known transparent scalar potential. It is found that the energy band edges are determined by a transcendental equation which is very similar to the corresponding equation for the Dirac Kronig-Penney model. The solutions of the Dirac equation are expressed in terms of elementary functions.
\end{abstract}

PACS numbers: 1315, 9440T

European Journal of Physics 24 (2003) 435-441

$\ddagger$ To whom correspondence should be addressed (boris@metodos.fam.cie.uva.es)

$\S$ On leave from Tomsk State University, 634050 Tomsk, Russia. 
New exactly solvable periodic potentials for the Dirac equation

\section{Introduction}

The properties of a relativistic particle in a one-dimensional periodic structure play an important role in understanding many phenomena in Solid State and Nuclear Physics [1] 2]. For this purpose the relativistic generalization of the classic Kronig-Penney model [2, 3, the numerical solution of the Dirac equation [1] and a class of exactly solvable nonlocal separable periodic potentials 4 4 have been studied. Nevertheless, due to its elementary character, the Dirac Kronig-Penney model remains the most frequently used model for studying one-dimensional relativistic band structures.

A new method for constructing exactly solvable non-relativistic periodic potentials has been recently proposed [6]. Starting from an easily solved (nonperiodic) potential, a new solvable potential is generated by using the familiar Darboux transformation. This new potential is then considered on a bounded interval and its periodic extension is constructed. In this paper we generalize this procedure to construct a solvable scalar potential for the Dirac equation.

\section{Band structure for a periodic potential}

Here we recall the main features of the Dirac equation with a periodic potential (see e.g. 2, 3]).

Consider the one-dimensional Dirac equation

$$
h \psi(x)=E \psi(x), \quad h=i \sigma_{y} \partial_{x}+V(x),
$$

where $V(x)$ is a real self-adjoint matrix potential, which we assume has the canonical representation (for more details see e.g. [5])

$$
V(x)=p(x) \sigma_{z}+q(x) \sigma_{x} .
$$

$\sigma_{x, y, z}$ are the usual Pauli matrices and $\psi(x)=\left(\psi_{1}(x), \psi_{2}(x)\right)^{t}$ (the superscript " $t$ " meaning the transpose) is a two-component spinor wave function. For any real value of the parameter $E$ equation (11) has two real linearly independent solutions $\psi(x)$ and $\varphi(x)$. If $E$ is chosen to be real, then the wave functions can be chosen real as well. The adjoint equation for $\varphi(x)$ is

$$
-i \partial_{x} \varphi^{+}(x) \sigma_{y}+\varphi^{+}(x) V(x)=E \varphi^{+}(x) .
$$

If we multiply (11) by the row-vector $\varphi^{+}(x)$ from the left and (3) by the column-vector $\psi(x)$ from the right, the difference takes the form $[W(\varphi, \psi)]_{x}=0$ (the subscript $x$ stands for the derivative with respect to $x$ ) meaning that the function

$$
W(\varphi, \psi)=\varphi^{+}(x) i \sigma_{y} \psi(x) .
$$

is (a non-zero) constant. Hence, $W(\varphi, \psi)$ here plays the role of a Wronskian for the Dirac equation. Since $\psi$ and $\varphi$ form a basis set for the two-dimensional solution space of (1) for any given $E$, any solution of (1) with the same $E$ may be expressed as

$$
\chi(x)=A \psi(x)+B \varphi(x),
$$

where $A, B \in \mathbb{C}$. Without loss of generality we may assume that $\psi$ and $\varphi$ have been chosen so $W(\psi, \varphi)=1$.

Suppose now that $V(x)$ is a periodic potential:

$$
V(x)=V(x+T) \quad T \in \mathbb{R} .
$$


By Floquet's theorem, in this case (11) has Bloch solutions such that

$$
\chi\left(x_{0}\right)=\beta \chi\left(x_{0}+T\right) \quad \beta=\text { const } \in \mathbb{C},
$$

where $x_{0}$ is any point on the real axis. By using (5) we get the following equation for the coefficients $A$ and $B$ :

$$
\psi\left(x_{0}\right) A+\varphi\left(x_{0}\right) B=\beta\left[\psi\left(x_{0}+T\right) A+\varphi\left(x_{0}+T\right) B\right] .
$$

which is equivalent to the system of linear homogeneous equations:

$$
\begin{aligned}
& \left.\left[\psi_{1}\left(x_{0}\right)-\beta \psi_{1}\left(x_{0}+T\right)\right] A+\left[\varphi_{1}\left(x_{0}\right)-\beta \varphi_{1}\left(x_{0}+T\right)\right)\right] B=0 \\
& {\left[\psi_{2}\left(x_{0}\right)-\beta \psi_{2}\left(x_{0}+T\right)\right] A+\left[\varphi_{2}\left(x_{0}\right)-\beta \varphi_{2}\left(x_{0}+T\right)\right] B=0}
\end{aligned} .
$$

For such a system to have a non-trivial solution the determinant of coefficients must vanish, which is the case only if $\beta$ satisfies the quadratic equation

$$
\beta^{2}-D \beta+1=0,
$$

where

$$
D=W\left(\psi\left(x_{0}+T\right), \varphi\left(x_{0}\right)\right)+W\left(\psi\left(x_{0}\right), \varphi\left(x_{0}+T\right)\right)
$$

and we have used the property $W(\psi, \varphi)=1$. We get from (10) the two possible values for $\beta$

$$
\beta_{1,2}=D / 2 \pm \sqrt{D^{2} / 4-1}, \quad \beta_{1} \beta_{2}=1 .
$$

This result is quite similar to that for the Schrödinger equation with a periodic potential [6]. The function $D=D(E)$ is called the Lyapunov function or Hill determinant for the Dirac equation, and is real since $\psi$ and $\varphi$ are.

It is clear that for $|D(E)|<2$ the values of $\beta$ lie on the unit circle of the complex $\beta$-plane, so we can write them

$$
\beta_{1}=\exp (i K T), \quad \beta_{2}=\exp (-i K T) .
$$

with real $K$. It follows from (7) that the Bloch solutions are bounded on the whole real axis. Hence, all real $E$ with $|D(E)|<2$ lie in allowed bands. Since for $|D(E)|>2$, $\beta_{1,2}$ are real and positive, either Bloch solution is unbounded. All such $E$ form the forbidden bands, while the band edges are given by $|D(E)|=2$ and in general form the unbounded sequence

$$
\ldots E_{-4}<E_{-3} \leq E_{-2}<E_{-1}<E_{1}<E_{2} \leq E_{3}<E_{4} \ldots
$$

An important feature of the band structure for the Dirac equation is that allowed bands may exist for negative energy.

\section{Darboux transformation operator}

The Darboux transformation provides a very powerful method for finding new exactly solvable potentials, both for the Schrödinger equation [7] and the Dirac equation [8]. In this section we shall describe the main ideas for this method following [8, 9].

Consider the stationary one-dimensional Dirac equation for the Hamiltonian $h_{0}$ :

$$
h_{0} \psi(x)=\left(i \sigma_{y} \partial_{x}+V_{0}(x)\right) \psi(x)=E \psi(x) .
$$

Suppose the solutions of (15) are known for all $E$ and we wish to solve a second Dirac equation having Hamiltonian $h_{1}$ :

$$
h_{1} \varphi(x)=\left(i \sigma_{y} \partial_{x}+V_{1}(x)\right) \varphi(x)=E \varphi(x) .
$$


Instead of solving (16) directly, one looks for a transformation operator (or intertwiner) $L$ such that

$$
L h_{0}=h_{1} L .
$$

If such an operator can be found, then the eigenspinors of $h_{1}$ may be obtained by applying $L$ to those of $h_{0}: \varphi(x)=L \psi(x)$.

It has been shown (see [8, 9] for details) that a first order differential operator

$$
L=\partial_{x}-u^{\prime}(x) u^{-1}(x)
$$

satisfies (17) with

$$
V_{1}(x)=V_{0}+\left[i \sigma_{y}, u^{\prime}(x) u^{-1}(x)\right],
$$

where $u(x)=\left(u^{(1)}(x), u^{(2)}(x)\right)$ is a $2 \times 2$ matrix satisfying the equation

$$
h_{0} u(x)=u(x) \Lambda, \quad \Lambda=\operatorname{diag}\left(\lambda_{1}, \lambda_{2}\right) .
$$

It is easy to check that the spinors $u^{(j)}(x), j=1,2$ are eigenfunctions of the Hamiltonian $h_{0}$ corresponding to the eigenvalues $\lambda_{j}$. The matrix $u(x)$ is called a transformation function.

If $E \neq \lambda_{1}$ or $\lambda_{2}$, then by applying $L$ to any solution to (15), we obtain a corresponding solution to (16); if $E=\lambda_{1}$ or $\lambda_{2}$, then $L u^{(1,2)}(x)=0$. Nevertheless, in the latter case it can be shown that the function

$$
v(x)=\left(u^{t}(x)\right)^{-1}
$$

is a matrix eigenfunction for the Hamiltonian $h_{1}$ with eigenvalue matrix $\Lambda: h_{1} v(x)=$ $v(x) \Lambda$. Therefore the spinors $v^{(j)}(x), j=1,2$ are eigenfunctions of $h_{1}$ with eigenvalues $\lambda_{j}$. Solutions to $(16), \tilde{v}^{(1,2)}(x)$ for $E=\lambda_{1}$ and $E=\lambda_{2}$, may be found by means of the properties

$$
W\left(v^{(j)}(x), \tilde{v}^{(j)}(x)\right)=1, \quad j=1,2 .
$$

Therefore, with the help of the Darboux transformation one is able to obtain the solutions to the transformed equation (16) for any value of $E$ provided the solutions to the original equation (15) are known.

\section{Darboux transformation for a scalar potential}

A scalar potential is specified by a single function $p_{0}(x)=m+S_{0}(x), x \in \mathbb{R}$, so

$$
V_{0}=\left(m+S_{0}(x)\right) \sigma_{z}=\left(\begin{array}{cc}
m+S_{0}(x) & 0 \\
0 & -\left(m+S_{0}(x)\right)
\end{array}\right),
$$

where $m$ is the particle mass. For our purposes, it is more convenient to use the alternative form for the scalar potential

$$
\tilde{V}_{0}=\left(m+S_{0}(x)\right) \sigma_{x}=\left(\begin{array}{cc}
0 & m+S_{0}(x) \\
m+S_{0}(x) & 0
\end{array}\right) .
$$

These two potentials are related by the unitary transformation

$$
\tilde{V}_{0}=U^{-1} V_{0} U, \tilde{\psi}=U \psi,
$$

where

$$
U=\left(1+i \sigma_{y}\right) / \sqrt{2} \text {. }
$$


In general, when a Darboux transformation is applied to a scalar potential, the resulting potential is not scalar, but below we formulate additional conditions that will prevent this (see [8, 10] for details).

If the spinor $u^{(1)}=\left(u_{11}, u_{21}\right)^{t}$ is a solution to the Dirac equation (15) for the potential (24), for some energy $E=\lambda$, it is easy to check that the spinor $u^{(2)}=-\sigma_{z} u^{(1)}$ is a solution to the same equation for energy $E=-\lambda$. The transformation matrix $u(x)$ constructed from the spinors $u^{(1)}$ and $u^{(2)}$ can be seen to give

$$
u_{x} u^{-1}=\left(\begin{array}{cc}
\left(\ln u_{11}\right)^{\prime} & 0 \\
0 & \left(\ln u_{21}\right)^{\prime}
\end{array}\right),
$$

and the transformed potential (19) takes the form

$$
\begin{aligned}
& \tilde{V}_{1}=\left(m+S_{1}(x)\right) \sigma_{x}, \\
& S_{1}(x)=S_{0}+\left(\ln u_{21}\right)^{\prime}-\left(\ln u_{11}\right)^{\prime}
\end{aligned}
$$

which is clearly scalar.

With the help of (18) and (27) one finds the solutions to the transformed equation:

$$
\varphi=L \psi=\left(\begin{array}{c}
\psi_{1}^{\prime}-\left(\ln u_{11}\right)^{\prime} \psi_{1} \\
\psi_{2}^{\prime}-\left(\ln u_{21}\right)^{\prime} \psi_{2}
\end{array}\right) \text {. }
$$

Note that the components of this spinor are given by the same expressions that appear in the Darboux transformation, with transformation functions $u_{11}, u_{21}$, for the Schrödinger equation (see e.g. [1]).

\section{New periodic scalar potential}

The free particle Dirac Hamiltonian can be written in terms of the scalar potential (24) with $S_{0}=0$. One can easily verify that the spinors

$$
\begin{aligned}
& u^{(1)}(x)=(\cosh (\gamma x-\alpha), \cosh (\gamma x+\alpha))^{t}, \quad e^{2 \alpha}=\sqrt{\frac{m-\gamma}{m+\gamma}}, \\
& u^{(2)}(x)=-\sigma_{z} u^{(1)}(x)
\end{aligned}
$$

are eigenspinors of $h_{0}$ corresponding to the eigenvalues $\lambda_{1,2}= \pm \lambda= \pm \sqrt{m^{2}-\gamma^{2}}$. By constructing the transformation function from these spinors and using (29), we arrive at the reflectionless (one-soliton) potential corresponding to

$$
S_{1}(x)=-\frac{2 \gamma^{2}}{m+\lambda \cosh 2 \gamma x},
$$

which was found previously [12]. It is easily checked that the two spinors given by the matrix $\left(u^{+}\right)^{-1}$ are square integrable. Hence the potential (28), (32) has the two discrete levels $E= \pm \lambda$.

Linearly independent solutions of the Dirac equation for potential (32) found by means of (30) are

$$
\begin{aligned}
& \tilde{\psi}(x)=\frac{E}{\sqrt{\gamma^{2}+k^{2}}}\left(\begin{array}{l}
\cos k x-\frac{1}{k} w_{1}(x) \sin k x \\
\cos (k x-\delta)-\frac{1}{k} w_{2}(x) \sin (k x-\delta)
\end{array}\right), \\
& \tilde{\varphi}(x)=-\frac{1}{\sqrt{\gamma^{2}+k^{2}}}\left(\begin{array}{l}
k \sin k x+w_{1}(x) \cos k x \\
k \sin (k x-\delta)+w_{2}(x) \cos (k x-\delta)
\end{array}\right),
\end{aligned}
$$

where $k=\sqrt{E^{2}-m^{2}}, \delta=\arctan (k / m), w_{1,2}(x)=\gamma \tanh (\gamma x \mp \alpha)$. These are normalized such that their Wronskian is unity: $W\left(\tilde{\psi}_{E}(x), \tilde{\varphi}_{E}(x)\right)=1$. 


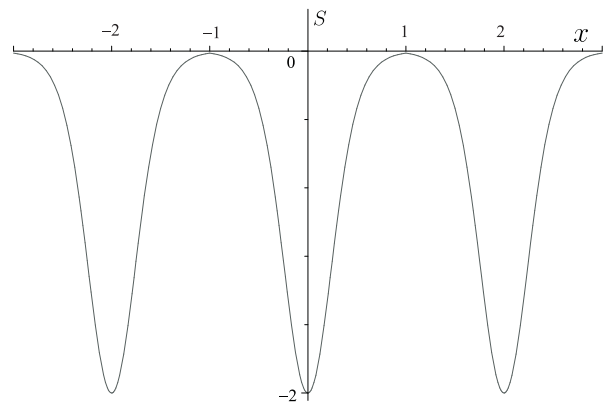

Figure 1: Periodically continued scalar potential.

Let us now restrict the potential as given by (32) to the interval $[-a, a], a>0$ and continue it to the entire $x$-axis by periodicity to obtain the continuous potential, having period $T=2 a$, shown in Fig. 1 for $m=2, \lambda=1$, and $a=1$. Having found the solution to the Dirac equation allows us to calculate the Lyapunov function (11). For this purpose we first have to calculate Wronskians at $x_{0}=-a$. So, using (33) and (34) after some simple algebra we find for the first one the expression:

$W(\tilde{\psi}(a), \tilde{\varphi}(-a))=\frac{E}{k^{2}+\gamma^{2}}\left[w_{1}(a)\left(\cos (2 k a+\delta)-\frac{1}{k} \cos (k a+\delta) \sin (k a) w_{1}(a)\right)\right.$

$\left.+k \cos (2 k a) \sin (\delta)-w_{2}(a)\left(\cos (2 k a-\delta)-\frac{1}{k} \cos (k a) \sin (k a-\delta) w_{2}(a)\right)\right]$

In the derivation we have used the symmetry relations $w_{1}(-a)=w_{2}(a), w_{2}(-a)=$ $w_{1}(a)$. After calculating the second Wronskian in (11) we obtain

$$
\begin{aligned}
D(E)=\frac{E}{k^{2}+\gamma^{2}} & {\left[2 w_{1}(a) \cos (2 k a+\delta)-2 w_{2}(a) \cos (2 k a-\delta)\right.} \\
+ & \left.\frac{1}{k}\left(k^{2}-w_{1}^{2}(a)\right) \sin (2 k a+\delta)-\frac{1}{k}\left(k^{2}-w_{2}^{2}(a)\right) \sin (2 k a-\delta)\right] .
\end{aligned}
$$

The behavior of this function is sketched in Fig.2

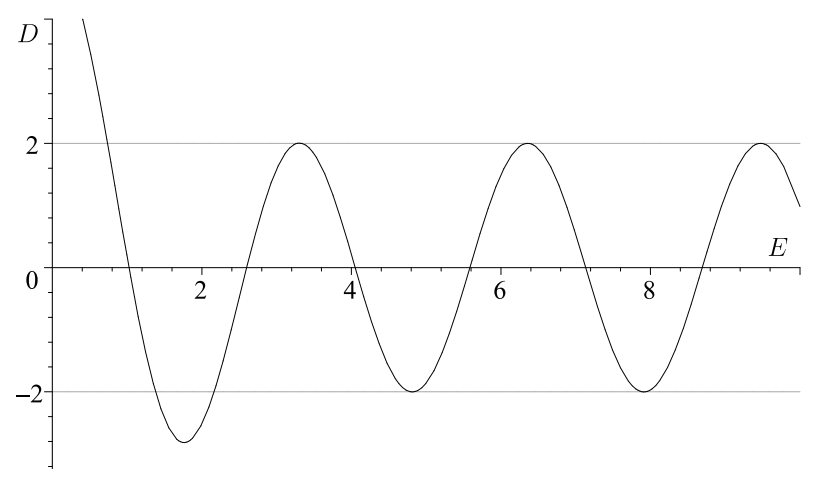

Figure 2: Lyapunov function for a periodic scalar potential.

for $m=2, \lambda=1$, and $a=1$ and leads to the following values for the lowest band edges in the positive spectrum: $E_{0}=0.738, E_{1}=1.381, E_{2}=2.164, E_{3}=3.274$, $E_{4}=3.335, E_{5}=4.802, E_{6}=4.827, E_{7}=6.352$. Since $D(E)$ is an even function of the energy, the negatives of these values form the band edges in the negative spectrum. 
We conclude this section by indicating how the actual energy bands may be constructed from our results. From (7), (11) and (13), since the Wronskian has the value unity, we see that

$$
\cos (2 K a)=\frac{1}{2} D(E)
$$

so that the wave vector $K$ is obtained as an explicit function of the energy in the $j$-th energy band by

$$
K=\frac{1}{2 a} \arccos \frac{1}{2} D(E) \quad \text { for } \quad E_{j-1}<E<E_{j} .
$$

By symmetry it is sufficient to consider only positive $K$, as we have implicitly done here. In general the inversion of this to get $E$ as a function of $K$ (the so called dispersion law) must be carried out numerically, but is trivially done graphically. The lowest positive energy band for the model above is shown in Figure 3.

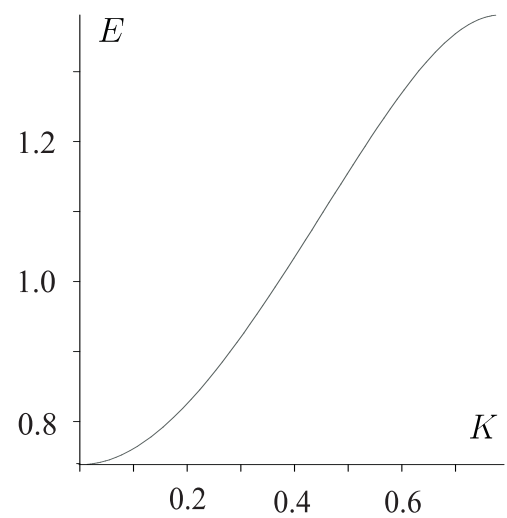

Figure 3: Lowest energy band for the case $\gamma=1, m=2$ for the scalar potential given by (32).

\section{Discussion}

In this note, using a specific example of one-soliton potential, we have shown how a great variety of exactly solvable continuous periodic local potentials for the one dimensional Dirac equation may be constructed. Furthermore, this procedure may be manipulated so that only elementary mathematical functions are involved in the construction and in the solution. Up till now, the only examples available have been simple variants of the classic Kronig-Penney model, yet this has been a key test bed in the relativistic theory of surface states [13], for example. We feel that the class of models presented here will be equally useful and lead to new insights in this and other areas.

\section{Acknowledgments}

The work of BFS was partially supported by the Spanish MCYT and the European FEDER (grant BFM2002-03773), and also by Ministerio de Educación, Cultura y Deporte of Spain (grant SAB2000-0240). MLG thanks the Universidad de Valladolid for hospitatlity and support. He also acknowledges partial support from the NSF (USA) under grant DMR-0121146. 


\section{References}

[1] Mendez B and Dominguez-Adame F 1991 J. Phys. A 24 L331

[2] McKellar B H J and Stephenson G J 1987 Phys. Rev. C 352262

[3] McKellar B H J and Stephenson G J 1987 Phys. Rev. A 362566

[4] Glasser M L 1983 Am. J. Phys. 51936

[5] Levitan B M and Sargsyan I S 1991 Sturm-Liouville and Dirac Operators Dordrecht: Kluwer

[6] Samsonov B F 2001 Eur. J. Phys. 22305

[7] Bagrov V G and Samsonov B F 1997 Phys. Part. Nucl. 28374

[8] Nieto L M, Pecheritsin A A and Samsonov B F 2003 Ann. Phys. 305 (2003) 151

[9] Samsonov B F and Pecheritsin A A 2000 Russ. Phys. J. 43(11) 48

[10] Samsonov B F and Pecheritsin A A 2002 Russ. Phys. J. 45(1) 74

[11] Bagrov V G and Samsonov B F 1995 Theor. Math. Phys. 104356

[12] Nogami Y and Toyama F M 1993 Phys. Rev. A 471708

[13] Davison S G and Steslicka M 1992 Basic Theory of Surface States Oxford: Clarendon Press 